\title{
Comment on: Morphologic Features of Buried Optic Disc Drusen on En Face Optical Coherence Tomography and Optical Coherence Tomography Angiography
}

In a well designed study Kim and associates ${ }^{1}$ have used en face OCT and OCTA to distinguish presumed cases of 'optic disc drusen' (ODD) from papilledema. Images presented are of excellent quality and permit to comment on the findings.

By definition, on OCT exposed ODD consist of a signal-poor lesion, which is occasionally associated with a hyper-reflective cap, horizontal hyperreflective lines or multiple aggregates. Buried ODD displayed the same OCT findings. The beautiful images presented do not resemble typical ODD as defined by consensus. $^{2}$

The images in present study show peripapillary hyper-reflective ovoid mass-like structure (PHOMS) (see Figure 2 in ${ }^{1}$ ). The authors have previously asserted that PHOMS are uncalcified precursors or variants of ODD. ${ }^{3,4}$ That "PHOMS are drusen" is a question that has been debated elsewhere and remains somewhat controversial. $^{2,4,5}$ The consensus report cautioned that unless there is histological confirmation, PHOMS should not be the basis for the diagnosis of ODD. ${ }^{2,5}$ Several of the coauthors have acknowledged the controversy noting "that if ODD are defined according to the.... [consensus] guidelines then PHOMS should be regarded as a different diagnostic entity". ${ }^{4}$ Regardless of the differing viewpoints, this report by Kim et.al. is an en face OCT study of PHOMS, not ODD.

For this communication, we would only point to the most compelling argument against equating PHOMS with drusen which is that PHOMS occurs in a variety of optic nerve head disorders. ${ }^{4}$ To underscore this point, we propose to define three categories of PHOMS: 1.Drusen-associated PHOMS would include cases that meet the consortium criteria for the OCT diagnosis of ODD with PHOMS. 2.Disc edemaassociated PHOMS includes patients with any type of disc edema and PHOMS, and 3.Tilted discassociated PHOMS seen in mild forms of the tilted optic disc or a myopic obliquely-inserted disc (MOID). This is a common cause of pseudopapilledema without drusen. It typically has an elevated, pale C-shaped halo nasally, retinal pigmentary changes temporally and unlike the fully developed tilted disc syndrome, has little or no rotation or retinal ectasia. Credit goes to Pichi et.al. ${ }^{6}$ who were first to note the frequent occurrence of a "dome-shaped hyper-reflective structure" nasally by OCT; identical to the PHOMS in ODD. ${ }^{2}$ Others have confirmed this observation. ${ }^{5}$

The patients with "buried drusen" in present study ${ }^{1}$ were myopic and remarkably similar to the "tilted discassociated PHOMS”.

The authors have raised an important point that should not be overshadowed by this controversy, which is that the underlying cause of PHOMS needs to be better understood. PHOMS is not a diagnosis; it is a nonspecific structural finding by OCT that elevates the optic disc. The composition of PHOMS must ultimately be determined histopathologically. There is some histopathology to support the view that PHOMS are herniating nerve fibers in papilledema and ODD. ${ }^{2,5}$ The authors of this paper have proposed a number of plausible alternatives, for example that PHOMS might due to granulation tissue or degenerating axons. ${ }^{4}$ Until this question is resolved we suggest using a descriptive nomenclature. 
1. Kim MS, Lee KM, Hwang JM, et al. Morphologic Features of Buried Optic Disc Drusen on En Face Optical Coherence Tomography and Optical Coherence Tomography Angiography. Am J Ophthalmol 2020.

2. Malmqvist L, Bursztyn L, Costello F, et al. The Optic Disc Drusen Studies Consortium Recommendations for Diagnosis of Optic Disc Drusen Using Optical Coherence Tomography. J Neuroophthalmol 2018;38(3):299-307.

3. Lee KM, Woo SJ, Hwang JM. Morphologic characteristics of optic nerve head drusen on spectraldomain optical coherence tomography. Am J Ophthalmol 2013;155(6):1139-47 e1.

4. Lee KM, Woo SJ, Hwang JM. Peripapillary Hyperreflective Ovoid Mass-Like Structures: Is It Optic Disc Drusen or Not? Letter to editor and author response. J Neuroophthalmol 2018;38(4):567-570.

5. Malmqvist L, Sibony PA, Fraser CL, et al. Peripapillary Ovoid Hyperreflectivity in Optic Disc Edema and Pseudopapilledema. Ophthalmology 2018;125(10):1662-4.

6. Pichi F, Romano S, Villani E, et al. Spectral-domain optical coherence tomography findings in pediatric tilted disc syndrome. Graefes Arch Clin Exp Ophthalmol 2014;252(10):1661-7. 